\title{
Whole Genome Sequences of the Tea Leaf Spot Pathogen Didymella segeticola
}

\author{
Yafeng Ren, ${ }^{1}$ Dongxue Li, ${ }^{1}$ Xiaozhen Zhao, ${ }^{1}$ Yong Wang, ${ }^{2}$ Xingtao Bao, ${ }^{1}$ Xue Wang, ${ }^{1}$ Xian Wu, \\ Delu Wang, ${ }^{3}$ Baoan Song, ${ }^{1, \dagger}$ and Zhuo Chen ${ }^{1, \dagger}$ \\ ${ }^{1}$ Key Laboratory of Green Pesticide and Agricultural Bioengineering, Ministry of Education, Guizhou \\ University, Guiyang, Guizhou 550025, China \\ ${ }^{2}$ College of Agriculture, Guizhou University, Guiyang, Guizhou 550025, China \\ ${ }^{3}$ College of Forestry, Guizhou University, Guiyang, Guizhou 550025, China
}

\begin{abstract}
The fungal pathogen Didymella segeticola (basionym Phoma segeticola) causes leaf spot on tea (Camellia sinensis), which leads to a loss in tea leaf production in Guizhou Province, China. $D$. segeticola isolate GZSQ-4 was sequenced using Illumina HiSeq and Pacific Biosciences (PacBio) RS technologies, and then assembled to approximately 33.4 Mbp with a scaffold N50 value of approximately 2.3 Mbp. In total, 10,893 genes were predicted using the Nonredundant, Gene Ontology, Clusters of Orthologous Groups, Kyoto Encyclopedia of Genes and Genomes, and SWISS-PROT databases. The whole-genome sequence of $D$. segeticola will provide a resource for future research on host-pathogen interactions, determination of trait-specific genes, pathogen evolution, and plant-host adaptation mechanisms.
\end{abstract}

\section{Genome Announcement}

Phoma spp. are significant phytopathogens and can cause diseases of several plant organs, including root, stem, and leaf, in a range of plant species. For instance, Phoma sorghina causes leaf spot of maize and wheat (Amaral et al. 2004; Perelló and Moreno 2005), Phoma sp. QC2014b (LC 1633) causes leaf spot of the Tibetan thistle (Cirsium segetum) (Chen et al. 2015), and $P$. bellidis causes stem blight of Eleocharis dulcis and leaf spot of Angelica dahurica ( $\mathrm{Lv}$ et al. 2011; Xu et al. 2016). According to the morphological characters of pycnidia, conidia, colonies, et cetera, Phoma spp. is known as a form genus. Phoma spp. comprises nine sections, which contain 223 species (Boerema et al. 2004). Nevertheless, Chen et al. (2017) classified 360 strains of the family Didymellaceae into 19 monophyletic generic clades by multilocus phylogenetic tree, and 9 sections in Phoma spp. were clustered into different monophyletic generic clades. Phoma segeticola was first reported by Chen et al. (2015) and can cause leaf spot on Tibetan thistle. $P$. segeticola was further clustered into monophyletic generic clades of Didymella. So, P. segeticola was renamed Didymella segeticola (Chen et al. 2017). Tea plants (Camellia sinensis) are widely grown in Guizhou Province, which has the greatest tea cultivation area (35× $10^{4}$ ha) among all the provinces or cities in China (Li et al. 2019; Zhao et al. 2018). Tea leaf spot caused by $D$. segeticola widely occurs in the tea plantations of Guizhou Province, which lead to a huge loss of tea leaves (Li et al. 2019; Zhao et al. 2018). Presently, 14 genomes in the family Didymellaceae at $\mathrm{NCBI}$ are available for use in studying host-pathogen interactions, important traits, and pathogen evolution and plant-host, except the genome of $D$. segeticola being reported in our research group.

Fourteen isolates of $D$. segeticola var. camelliae were collected from Shiqian County in Guizhou Province from May 2016 to November 2017 (Zhao et al. 2018). These morphological

${ }^{\dagger}$ Corresponding authors: Z. Chen; gychenzhou@aliyun.com, and B. A. Song; basong@gzu.edu.cn

The author(s) declare no conflict of interest.

Accepted for publication 7 June 2019.

\section{Funding}

This project was supported by National Key Research Development Program of China (2017YFD0200308), the Major Science and Technology Projects in Guizhou Province (2012-6012), the Program for New Century Excellent Talents in University (NCET-13-0748). 
Table 1. Genome assembly statistics of Didymella segeticola isolate GZSQ-4

$\begin{array}{lr}\text { Statistics } & \text { Isolate GZSQ-4 } \\ \text { Assembly } & 23 \\ \text { Number of all scaffolds } & 33,448,292 \\ \text { Bases in all scaffolds } & 23 \\ \text { Number of large scaffolds (>1,000 bp) } & 33,448,292 \\ \text { Bases in large scaffolds } & 3,976,106 \\ \text { Largest length } & 2,254,797 \\ \text { Scaffold N50 } & 998,506 \\ \text { Scaffold N90 } & 51.85 \\ \text { G+C content (\%) } & 0 \\ \text { N rate (\%) } & 10,893 \\ \text { Gene prediction } & 17,069,577 \\ \text { Gene number } & 1,566 \\ \text { Gene total length (bp) } & 0.325 \\ \text { Gene average length (bp) } & 55.40 \\ \text { Gene density (kb) } & 51.0 \\ \text { GC content in gene region (\%) } & \\ \text { Gene/genome (\%) }\end{array}$

characteristics were similar with $D$. segeticola described by Chen et al. (2017). In the multilocus phylogenic analysis, the strain GZSQ-4 was identical to reference strains $D$. segeticola CGMCC 3.17489, and the clade was supported by $99.89 \%$ bootstrap values. Based on Koch's postulate, these isolates proved to be the causal agents of tea leaf spot (Zhao et al. 2018). Genomic DNA was extracted from $D$. segeticola isolate GZSQ-4 using fungal miniprep kits (E.Z.N.A.; Omega Bio-Tek, Inc.). A DNA library with fragment sizes of approximately 300 to 500 bp was prepared using a TruSeq DNA Sample Prep Kit. Bridge-PCR, on the Illumina HiSeq platform, was conducted using a TruSeq PE Cluster Kit and the products were then sequenced using a TruSeq SBS Kit with the Illumina HiSeq PE technology, generating $53,530,422$ raw reads and $8,029,563,300$ raw bp. The Illumina reads were trimmed, corrected, and filtered. A total of 51,270,673 clean reads and 7,598,831,045 clean bp passed the quality control criteria and were used in subsequent analyses of $D$. segeticola isolate GZSQ-4. There was $227 \times$ average coverage. Meanwhile, a Pacific Biosciences (PacBio) RS library, having fragment sizes of approximately 8,000 to $10,000 \mathrm{bp}$, was prepared using G-tubes and sequenced using single-molecule real-time cells, generating PacBio reads. PacBio reads were further filtered, generating PacBio subreads, with 1,489,032 total reads, and $11,821,890,861$ total bp. The largest length was $63,621 \mathrm{bp}$, and the N50, N90, and average lengths were $10,453,5,327$, and 7,939 bp, respectively. The genome size was $\sim 36.7 \mathrm{Mbp}$ based on read sequence data being processed with the condition of 21 -mers using the K-mer statistical method.

The raw sequence data were assembled using SOAPdenovo v2.04 (http://www.genomics.cn/) and then treated using GapCloser v1.12. The whole-genome assembly was $\sim 33.4 \mathrm{Mbp}$, with a scaffold N50 value of $\sim 2.3 \mathrm{Mbp}$ (Table 1). The gene prediction was annotated using Nonredundant (Nr), Gene Ontology (GO), Clusters of Orthologous Genes (COG), Kyoto Encyclopedia of Genes and Genomes (KEGG), and SWISS-PROT databases using BLAST 2.2.28+, resulting in 10,893 annotated genes. For instance, the COG analysis was conducted using the eggNOG database v4.5.1 (http://eggnogdb.embl.de/\#/app/home), and a KEGG enrichment analysis was also conducted (https://www.genome.jp/kegg/). The GO database was used to annotate genes at the cellular component, molecular function, and biological process levels (http://geneontology.org/). D. segeticola showed a high abundance of GO terms for catalytic activity (890), binding (628), transporter activity (113), and structural molecule activity (18).

The genome sequences of $D$. segeticola presented here provide a unique resource for studying pathogenicity determinants in this pathogen, understanding mechanisms of host adaptation, and designing specific disease control management strategies. The genome sequence has been deposited in GenBank under genome accession SDAV00000000 (BioProject PRJNA516041; BioSample SAMN10768991; and SRA accession SRR8492863). 


\section{Literature Cited}

Amaral, A. L. D., Carli, M. L. D., Neto, J. F. B., and Soglio, F. K. D. 2004. Phoma sorghina, a new pathogen associated with Phaeosphaeria leaf spot on maize in Brazil. Plant Pathol. 53:259.

Boerema, G. H., Gruyter, J., Noordeloos, M. E., and Hamers, M. E. C. 2004. Phoma Identification Manual. Differentiation of Specific and Infra-Specific Taxa in Culture. CABI Publishing, Wallingford.

Chen, Q., Hou, L. W., Duan, W. J., Crous, P. W., and Cai, L. 2017. Didymellaceae revisited. Stud. Mycol. 87:105-159.

Chen, Q., Zhang, K., Zhang, G. Z., and Cai, L. 2015. A polyphasic approach to characterize two novel species of Phoma (Didymellaceae) from China. Phytotaxa 197:267-281.
Li, D. X., Bao, X. T., Ren, Y. F., Wang, Y., Song, B. A., and Chen, Z. 2019. First report of Lasiodiplodia theobromae causing leaf spot on tea plant in Guizhou province of China. Plant Dis. 103:374.

Lv, R., Zheng, L., Zhu, Z., Pan, L., Huang, J., and Hsiang, T. 2011. First report of stem blight of Eleocharis dulcis caused by Phoma bellidis in China. Plant Dis. 95:1190.

Perelló, A. E., and Moreno, M. V. 2005. First report of Phoma sorghina (Sacc.) Boerema Dorenbosch \& van Kest on wheat leaves (Triticum aestivum L.) in Argentina. Mycopathologia 159:75-78.

Xu, H. J., Cui, J. C., Zhou, R. J., Fu, J. F., and Hao, N. 2016. First report of leaf spot disease in Angelica dahurica caused by Phoma bellidis in China. J. Phytopathol. 164:448-454.

Zhao, X. Z., Wang, Y., Li, D. X., Ren, Y. F., and Chen, Z. 2018. Morphological characterization and phylogenetic analysis of the pathogen Phoma segeticola var. camelliae causing a new tea disease. Acta Phytopathol. Sin. 48:556-559. 